\title{
Building Educational Simulations Using KSA Open Data
}

\author{
PATRICIA MARYBELLE DAVIES \\ College of Sciences and Human Studies \\ Prince Mohammad bin Fahd University \\ Al Khobar \\ KINGDOM OF SAUDI ARABIA
}

\author{
PHIL WEIR, SARAH BYRNE \\ Flax \& Teal Limited \\ Farset Labs, Belfast \\ Northern Ireland \\ UNITED KINGDOM
}

\begin{abstract}
This paper reports on the development of ORP-Sandstorms, an open-source, web-based simulation which provides students with virtual experiences of the impact of global natural disasters, such as earthquakes and sandstorms. The project is a collaboration between an open-software developer and an educational technology researcher. The simulation utilizes a number of open datasets, including those available in the Saudi Open Data portal, to estimate the impact of such natural events on familiar local landmarks. Open data allows contextualization of abstract concepts in a local setting, to enable students to relate more easily. Understanding the impacts and benefits of this contextualization in the Kingdom of Saudi Arabia (KSA) context will form a core component of the present educational research. The KSA open data is crucial for testing adaptability to new regions and communities. A key outcome of this work is documenting the adaptability achievements and challenges of taking the established OurRagingPlanet platform, developed mainly from Northern Ireland datasets, and leveraging novel uses of KSA open data to re-contextualize ORP-Sandstorms for KSA students.
\end{abstract}

Key-Words: - educational simulations, KSA open data, Arabic translations, Weblate, gaze detection, text tagging

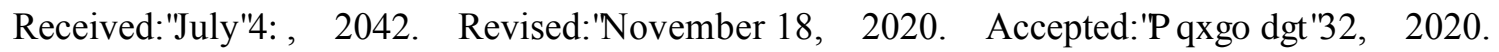
Published: December 8, 2020.

\section{Introduction}

Over the past two decades, games and simulations have been used in education and business to enhance learning [1]. A simulation is a dynamic, valid, simplified scenario representing reality that is implemented electronically [文]. Unlike games, simulations do not involve competition. The benefits of simulations include that they allow users to encounter problem situations, formulate decisions and take actions, experience the results, and then modify their behaviour without risking harm. Open data - digital content that is freely available, and can be modified and shared by anyone for any purpose [3] - is central to the databased fourth industrial revolution. Typically provided by governments and public organizations, open data facilitates transparency and deeper interaction with stakeholders, and also helps realize social and economic value. Open government data is perhaps the most important form of open data. These are generated through constant interactions and activities, including internet communications, banking transac- tions, and mobile phone use, which form part of the daily lives of their citizens.

The web application, ORP-Sandstorms, features custom tools and interactive maps that allow users to apply basic simulations to their local area. It utilizes open data to provide more realistic estimates of the impact natural disasters can have on familiar local landmarks within the coverage area. Part of the application simulates sandstorms as they occur on the Arabian Peninsula, and is developed using datasets from the data.gov.uk portal relevant to Northern Ireland, including railways stations, sports fields and elevation data. The other simulations utilize KSA Open Data.

\section{Purpose}

The purpose of the work reported in this paper is research into and the development of ORP-Sandstorms, a web-based educational simulation designed to promote understanding of natural disasters and their impact. Schools and colleges within the Kingdom of Saudi Arabia (KSA) and in the United Kingdom (UK) 
will use ORP-sandstorms to access simulations of natural events different from those they encounter locally. Specifically, KSA students will experience storm surges while students in the UK will simulate sandstorms. The aim is to develop in these learners an understanding of the natural world and empathy for other cultures, in addition to critical thinking skills and a need for resilience. Through the simulations, students will learn to relate to natural disasters happening elsewhere, thereby developing deeper understandings of some of the ideas, customs, and social behaviours of people living in other regions. Additionally, ORP-Sandstorms highlights the physical basis and limitations of such events, thus making it an excellent tool for teaching students about geological events in a holistic manner.

The simulations are technical components of an empirical research project by the same name designed to test the effectiveness of simulation tools on modern network-based platforms that support learning with technology. The research also aims to collect and analyze data examining how and why ORP-Sandstorms could extend learners' understandings of the natural world and of other cultures beyond what they would typically see in television or online news reports. This is an attempt to further pedagogical understandings of immersive virtual reality educational applications which, as Bower and Jong [4] suggest, lag behind their technological developments. The expected outcome of the empirical research is a pedagogical model and enabling tools for the design and integration of simulation software that supports learning [5, 6, 7]]. In addition, the simulations are aimed at enabling students to empathize with the experiences and concerns of their contemporaries around the world, with a focus on educating the students in the UK about the environmental challenges experienced by people living in KSA and vice versa.

The segment of the software available to KSA students will simulate disasters such as volcanoes and storm surges, which typically do not occur in KSA, happening locally. The other segment, available to UK students, will include simulations of sandstorms - a regular occurrence in KSA, yet uncommon in the UK - happening within their local community. The interface is tailored to simplify use by students and teachers. ORP-Sandstorms will enable quick set-up and minimize in-classroom preparation time. The learning resource will be accessible internally and externally, to facilitate use in lesson plans for delivering relevant parts of the National Curriculum in schools in both countries. Therefore, the research project goes beyond the technical aspects of software development. It facilitates international collaboration between academics and technology experts, and promotes utilizing local maps to contextual- ize learning while furthering social science research.

\section{Background}

For a number of decades, simulations have been utilized for finding solutions to real-world problems in areas such as medicine and aviation, where it is not possible to rehearse due to logistical challenges, danger, or high costs [8, 9]. It was not until the 1970s and 1980s that simulations began to take advantage of digital technologies and have increasingly relied on them to provide learners with a more flexible real worldview, increased access, and detailed feedback. Their advantages for learners include making it possible to achieve specific learning objectives through repetition and practice without risk or harm, and facilitating a trial-and-error approach, often without penalty. Outcomes can be measured using validated scoring systems. These scores, combined with reflective exercises [10], can serve as feedback for a formative assessment cycle of repeated performance practice and improvement [11] in skills development exercises.

The Open Government Initiative was started under President Barack Obama in January 2009 [12]. The rationale underpinning the initiative centers on the fact that technology has grown at a rapid pace over the past two decades and this has changed the amounts of, and ways in which, we share information. So too has the demand for transparency by governments about how they create legislation and make policy decisions. As a result, the US created Open Government Data - first, to make freely available to the general public information that is being used to represent them; second, to enable the private sector to utilize this data for the common good. Many governments around the world have since established similar datasets. There are now open data indices measuring the readiness, implementation and emerging impact of an open government data portal.

The Open Data movement is a very significant and potentially powerful emerging force. Endorsed by the father of the World Wide Web, Tim Berners-Lee, open data make available data acquired publiclylocal, regional and national - in formats that allow direct software manipulation for different purposes including, mapping, cross-tabulation, and visualization. The idea of public and private data being made publicly available digitally and accessible via the Web, is a drive towards increased transparency that allows for an enhanced data-driven citizen or public engagement in policy and other analysis and assessment. It does not matter if this data is collected directly-for example, during a census - or as secondary output from other sources such as statistics relating to hospitalization, accidents and crime.

Part of the innovation in ORP-Sandstorms brings together several elements of the Science and Geog- 
raphy syllabuses that some students may find hard to relate to, in a personal way by contextualizing local data. All of this is made possible through the use of KSA Open data, which features datasets about the economy, education, employment and social development, environment, finance, health, traffic accidents, transport and weather in Saudi Arabia. This important KSA initiative is a strategy for transparency, public participation and innovation. The portal publishes datasets from ministries and government agencies in an open format in an attempt to bridge the gap between governments and citizens. The KSA Open Data portal helps clarify the work of government agencies, and provides opportunities for citizens to evaluate the performance of such institutions in order to make informed decisions about policies [13].

The ORP-Sandstorms project resonates with KSA's development goals. Saudi Arabia's Vision 2030 reform suggests that education should be centered on improving students' skill sets and confidence, and promote critical thinking [14]. Vision 2030 also seeks to showcase the Kingdom's rich culture, heritage and traditions to the world and to encourage cultural exchange. ORP-Sandstorms would be an important step in achieving these goals and, as such, could have a strong societal impact.

\subsection{The Simulation}

ORP-Sandstorms extends an existing web-based application called OurRagingPlanet (ORP), which is open source software developed by Flax \& Teal. It is designed for technical and non-technical users, using a visual and interactive approach. Users can generate local landscapes and environmental hazards, and incorporate these into a simulation of a natural disaster in that region. Existing ORP simulations of flash floods and volcanoes, are modified to fit the Saudi Arabian context. Inclusive of this is translating existing content from English to Arabic. The simulations are made available via the web, as shown in Figure 1. Environments are created using an admin panel. Based on the chosen region and location, available geospatial open data are dynamically excised for inclusion or exclusion. The simulations can then be composed based on the specific location and data region.

The main complexity in developing the platform exists in structuring simulation definitions so that new simulation models, such as the ORP-Sandstorms sandstorm functionality, can be added with any necessary parameters and defining environmental and data requirements that the system can automatically validate and inject. This is in contrast to static systems where a developer would need to modify the system to add a simulation, such as a given sandstorm algorithm, to the code, or tie it to one specific ge- ographical or data region and fixed known formats. The object toolbox provides simplified, studentfriendly layer management- primarily, enabling and disabling open dataset visibility-allowing users to experiment in a layout representative of their real, local environment.

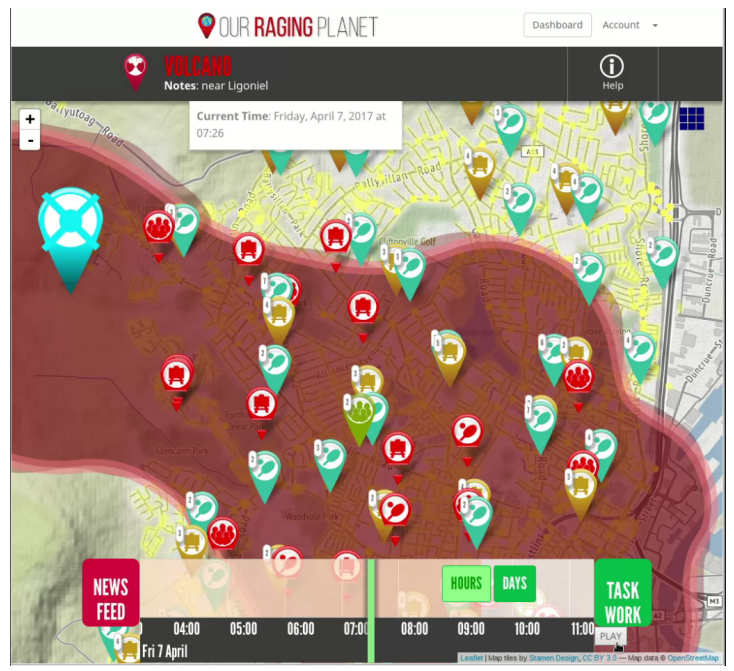

Figure 1: Screenshot of the OurRagingPlanet interface

Additional features relevant to the education field, intended to further enhance the immersiveness of experience, are the News Feed, for manually attaching imagined social media or news posts to the timeline, and the Task Work feature, for similarly tagging quiz questions or other exploratory tasks at specific points in the simulation.

\section{Datasets Used in the Simulations}

This section summarizes the main datasets that make it possible for the simulations to recreate familiar local KSA landmarks. Our approach has been to combine the event simulation with the KSA and other open data to increase the potential of data generation and sharing, thereby making it possible for us to achieve our objectives.

\subsection{Geospatial data}

Using open data from Copernicus, European Union's Earth Observation Programme, in conjunction with Sentinel Online, we can utilize satellite image data to populate elevation, waterways and agricultural land. Roadways and urban areas are populated from data available on OpenStreetMap, an open source map web application. Integrating such data is done using the platform CKAN, a data management system that provides tools to streamline publishing, sharing, searching and using data. In this development, CKAN is used in the backend of the OurRagingPlanet platform. 


\subsection{KSA Open Data}

The Kingdom of Saudi Arabia's open data portal is a vital source of data for urban and rural assets. For example, it provides information on all public wells, agricultural land, railways, location and number of hospitals and academic institutions in KSA. This data can be used to build a more accurate picture of the urban landscape and allow learners to contextualize the simulation since it can be placed within their regional context. However, despite the abundance of open data available for the Kingdom of Saudi Arabia, many of these datasets do not have latitude and longitude geospatial information. This is necessary information for populating the map base with more assets, to create a realistic sense of place for the learner. Therefore, the amount of local landmarks we can add is limited, in direct contrast to the wealth of geospatial UK data.

Information about sandstorms recorded in KSA Open Data, a useful context for educational simulations of sandstorms in the region, is also limited. What exists are the number of sandstorms that occurred within a given month or day by individual province, recorded by the Presidency of Meteorology and Environment for the periods 1999-2001 and 2003-2012. This data could help identify the areas most vulnerable to sandstorms, and is, therefore, a necessary feature for the simulation.

\subsection{UN Sandstorms Data}

The United Nations holds open data on dust storm days historically across Saudi Arabia, Egypt, Turkmenistan, Israel and Kuwait [15]. The data extends from 1961-1990 but only supplies the mean number of dust storm days in each region. The datasets provide important historical context for the frequency of dust storms in the Arabian Peninsula.

\subsection{Non-open Data}

We utilize additional platforms providing information in the form of real-time monitoring and visualisation of aerosol atmospheric data in the Arabian Peninsula. The North African Sandstorm Survey generates day and night remote sensing images in order to detect sandstorms over the Sahara and Saudi Arabia, and estimate day and night aerosol optical depth [16]. This open-public database contains information from 2004 to present. The Masdar Institute in Khalifa University in the United Arab Emirates has produced a realtime atmospheric composition forecasting web-app and wind atlas [17], which is useful in understanding how accurate forecasting of dust events and sandstorms is achieved to better prepare people and sectors in an attempt to minimize damage and loss of life.

We have been challenged by the limited availability in the KSA Open Data. We were hoping to find at least similar matches to the datasets in the OpenDataNI [18]. Instead, what exists in KSA Open datasets appear to serve functional purposes by recording services and quantities available per region for the KSA government. Table 1 shows a comparison of some of the datasets used in the OurRagingPlanet simulations with ones available in KSA Open Data. As noted by Al Rushaid and Saudagar [19], such discrepancies can be attributed to the fact that the public sector in KSA remain uninvolved in the initiative. It is also apparent that open data driven by government policies gain better recognition. Unfortunately, this is not currently the case in KSA.

The Open Data Barometer [20] captures this difference in the general openness of the two open government data portals by assigning the UK an index of 76 while KSA has an index of 25 . An interesting fact considering that KSA launched its open data portal in 2011, two years after the UK launched theirs. Projects like ours also serve to raise awareness of the need to extend open data portals by KSA and other governments.

\section{Developing the Simulation}

In this section, we discuss some of the technical aspects of the platform, including, its architecture, the underlying framework, how translating into Arabic works and planned prospects. Also discussed are some of the challenges encountered in the development and how these were overcome.

\subsection{Definitions}

ASC core: Abstract-Simulation-Case is a division of the domain model into separate areas of concern, providing interchangeability boundaries.

Abstract layer: a set of domain entities representing relationships between dynamically-defined numerical models and phenomena, parameter types, lists and matching rules.

Case context: a concretization of geophysical location, time and realization of extrinsic parameters.

Simulation: the application of an abstract model to a case context and realization of intrinsic parameters.

\subsection{Architecture}

The OurRagingPlanet platform uses BFF (Backend for Frontend) single-page-application. In the backend we are using Laravel and Twill for the admin panel, allowing some of the functionalities to compile in the backend then render on the frontend component. Laravel applications provide better performance than applications built with other frameworks due to the 
Table 1: Comparison of typical datasets in the UK OpenDataNI portal with those in KSA Open Data

\begin{tabular}{|l|l|}
\hline OpenDataNI & KSA Open Data \\
\hline City Parks & Park Locations \\
Community Centres & Health Education Activities \\
Historic Parks and Gardens & Tourism demand trips \\
Library Locations in Northern Ireland & National Training Institutes \\
Lighting Assets & Sewage Network Statistics \\
Northern Ireland Railways Stations & Railways \\
Playgrounds & No. of Water Subscribers, by region \\
Schools & No. of Hospitals and Beds, by region \\
\hline
\end{tabular}

caching system. The file cache driver stores items in the file system, alongside a dockerized redis cache for dynamic values, allowing for fast application development. It provides a simple and efficient authentication system and access controls, out-of-the-box, allowing you to restrict the access of unauthorized users to certain resources. Additionally, it provides application security, as the framework's codebase is protected from threats such as SQL injection or cross-site request forgery (CSRF), protecting loss of user data. This framework has a built-in Eloquent ORM system, which allows you to work with different databases, utilizing the implementation of the ActiveRecord pattern. With Eloquent ORM, you can work with chosen databases without having to write complex SQL queries. Indeed, Laravel supports MVC architectural design, as we are able to separate the user interface and business logic. It also supports the Blade templating engine, which allows native PHP code to be used. This is deployed onto a Kubernetes cluster together with disaster simulation serverless functions, using OpenFaaS. Twill is Laravel based framework which has helped us to build our custom CMS (Content Management System) that is user friendly, powerful, and dynamic. This framework has pre-built features and associated library of Vue.js UI components, allowing the developer to focus on the special needs of their application.

The frontend developed with Vue.js, backend and frontend connected with Nginx service which Vue.js allows you to split your application or page into logical units that can be used as reusable components. Each of these components can contain HTML, CSS and JavaScript code, which is inherent only to this component and is responsible for its performance. This framework is a solid JavaScript library with its own ecosystem that allows you to easily scale the application being developed. Vue.js is defined as ViewModel layer of MVVM template. It connects the model and view to two-way data binding. Current VDOM changes and formatted output are abstracted into Directives and Filters. Using VDOM completely separates possible DOM manipulations with the func- tional layer - now the function code works declaratively by composing VNodes, which makes it easier to maintain and add new functions.

\subsection{Model Interchange}

For the KSA context, several modifications to the interface are required for localization, to facilitate testing in an educational setting. These changes form a base for on-going educational research. However, due to the design of the underlying framework, dynamically adding first or third party sandstorm simulation, or re-imagining volcano simulation within $\mathrm{KSA}$, is possible without redesigning the framework or creating custom interfaces. The actual algorithm implementation is run in a containerized image, which allows for a wide variety of languages, run times and libraries to be used. The simulation process is scheduled using the OpenFaaS [21] serverless function framework, running over Kubernetes [22]. Simulation libraries that have been incorporated into various numerical model implementations include FEniCS [23] and Landlab [24].

\subsection{Arabic Translation}

OurRagingPlanet (ORP) first had to be translated into Arabic. For this, we used Weblate [25], a webbased translation tool that supports git-integration and a range of file formats. It offers all the features for professional translation work and also gives version control support. In Arabic, both the text and the entire page layout spread from right to left. As such, images that indicate direction need to be flipped. The direction attribute tells the browser the direction of text in an element. The global direction of the page must therefore be set using the direction attribute for the entire page. This presented the most challenging aspect of developing Arabic functionality within the platform.

The first step in the translation process was to prepare Weblate and the git repository for the integration. There are several ways to integration between Weblate and git, but the most suitable for us was using a translation repository as a submodule. Before starting 
the configuration for Weblate, a git repository to synchronize translations was prepared. For accessibility, we used the SSH key-also generated by Weblateand appended a "Weblate" user to our project with "Maintainer" permission. This kind of approach allows us to avoid merge conflicts. If we were to integrate our backend and frontend repository, we would have to add this user to each of them and configure the SSH key. A Weblate server can host many projects, each of which can have separate translatable components. In our case, we have a single ORP project in our weblate instance, with frontend and backend components. However, an inherent problem with using this structure is that translations will take place separately in each component, which means a translator would need to translate each word twice, once in the backend and once in the frontend. Therefore, one of the benefits of using the submodule was that it allowed us to use the "ORP project", as well as, one "ORP localization" component.

The second step in the process was to build an infrastructure for localization support. We decided to use GNU Gettext format, which has many advantages when used in Weblate. For example, it allows us to build bilingual translation, as well as plurals. Therefore, we have to append plugins for Laravel (backend) and Vue (frontend) as neither of them support ".po" file extension. In the backend, we used the "laravel-gettext" node module and for the frontend, we used "vue-gettext". Laravel-gettext does not have the functionality to extract texts from blade templates which is why we write our own console command with "PhpScanner", "BladeCompiler" and "PoGenerater" support. The "BladeCompiler" helps us to generate php files from blade template and use it in the "PhpScanner". The PhpScanner module can find tags (" ",", i"), as mentioned in templates. Then the "PoGenerator" builds ".po" files for translation. As "vuegettext" does not support the extracting strings from a project, we used an "easygettext" framework in the frontend. "Easygettext" can install general node modules and has commands which extract strings from Vue components and compile it to ".json" file. This .json file, used for translation is defined in the project as "main.js".

Third, we needed to merge the two word and annotations lists (.pot) into one file for Weblate and create continuous integration. Merge operation can be done with the "msgcat" command, however, in the folder level we must define the same location for both. The most appropriate place was a folder in the submodule as it allows to us easily access and synchronize the .pot files.

\subsection{Possibilities and Challenges}

The main novelty of the underlying framework for interchangeable simulations is its ability to flexibly interchange geophysical simulations with a high degree of domain independence. Many platforms exist that are able to dynamically interchange code components, often graphically; for example, [26, 27, 28]. However, this is insufficient to provide a nontechnical, seamless, unified interface, for creating interactive simulations using third-party numerical models over fourth-party data. Thus, a consistent set of abstractions must be established to build simple, non-technical interfaces. This must also capture essential behaviours across an unforeknown set of theoretical phenomena and numerical models, without restricting the ability to apply them to new geophysical settings and contextual data. The OpenAugury open source base, underpinning ORP, implements such a framework.

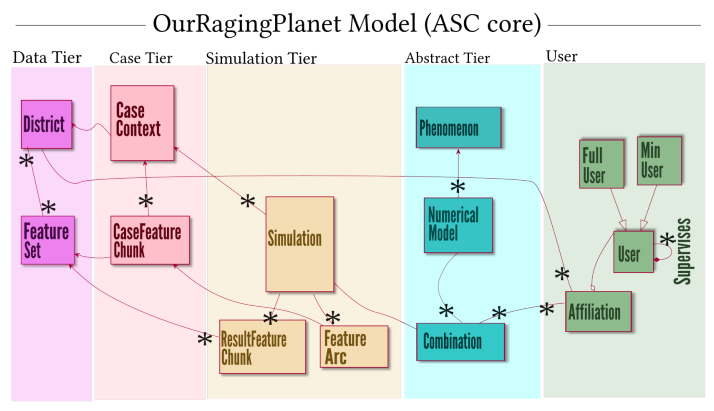

Figure 2: Simplified summary of key domain entities

The OpenAugury framework comprises two primary facets: a highly-adaptable simulation domain model (see Figure 2) and a compatible technical architecture. This structure draws on work performed in a clinical context [29] to create a clinicianfacing, model-independent simulation framework for multi-modal cancer treatments [30, 31]. The present work is a case study of the existing OpenAugury framework - built with support from OpenDataNIapplied to the KSA context and open data, thereby demonstrating the framework's adaptability, to allow the dynamic addition of physical phenomena, geographic settings, data sources in a highly contrasting context.

The development of ORP-Sandstorms posed several technical challenges, particularly when integrating KSA open data and localization to the KSA context. Here we highlight a few of these challenges, and how we have managed to work around them. Also discussed are some of the wider technical challenges posed by the platform. Figure 3 shows a phenomenon being simulated on the map. It includes a set of loca- 
tions with open data being presented, to provide context for students by presenting the environment at the location where the natural disaster is occurring. The areas in blue show simplified levels of simulation output, produced by dockerized simulators, in the background, providing long-running serverless output to generate the necessary data.

We had to think carefully about the interactions with a local context. Starting with the interface, it was necessary to ensure that the underlying map could also update to facilitate switching back and forth between Arabic and English. This is achieved by working with the Mapbox Studio tools and LeafletJS. Technical challenges arose around the implementation of the actual data translation. Where possible, we worked with human translators. For the technical data such as labels, we can rely to a certain degree on machine translations which have to be integrated. These localization settings need to update throughout the stack so that in changing the interface, the mapping layer, the simulation layer and the data layer get updated simultaneously. This was particularly challenging given the right-to-left nature of the Arabic language.

Running underneath are complex interactions of domain-level entities, broken into a series of domain layers framed for interchangeable simulation, known as the ASC core. Within a standard context not involving localization, this information is fed into data layers where we can then describe a particular context of that environment or datasets. To create ORP simulations, a case-context from the case tier is neededthis encapsulates the environment of an event, available geospatial data and the time frame. Thus the open data is used to create output representing the situation. The simulation tier then encompasses entities describing the event as it occurs within the simulation; something which is contextualised by the open data that is pulled into the simulation layer. A strength of the ORP platform is that it abstracts the simulation and context to enable the localization elements to be handled at a higher level. Therefore, the simulation model can be submitted by a numerical modeller without requiring them to, in many cases, factor in localization at all - this could even be done dynamically through the web platform. It can then be pulled in and used by existing data or new data, for existing users or new users, and swapped in and out depending on the desired context. This thereby provides a well-defined level of domain-level segregation. Thus, the ORP platform makes it possible for a numerical model based on somewhere else, such as the United States or Japan, to still be useable in Saudi Arabia, and vice-versa.

Challenges relating to the KSA open datasets include that many of them are in Arabic. In this case, we have utilized machine translations especially in cases of categorical data where these are non-contextual descriptive text. There is also a dearth of latitude and longitude data. We have looked at incorporating certain OpenStreetMap data to compensate, but ideally greater public data from KSA official sources would be preferred. Another element of localization presents itself here - data is often in local coordinate systems, for instance in Northern Ireland (our other test region), where the frame of reference for local data is the Irish grid. This means data must be converted to and from global coordinate systems transparently, to prevent this being a concern of the numerical model.

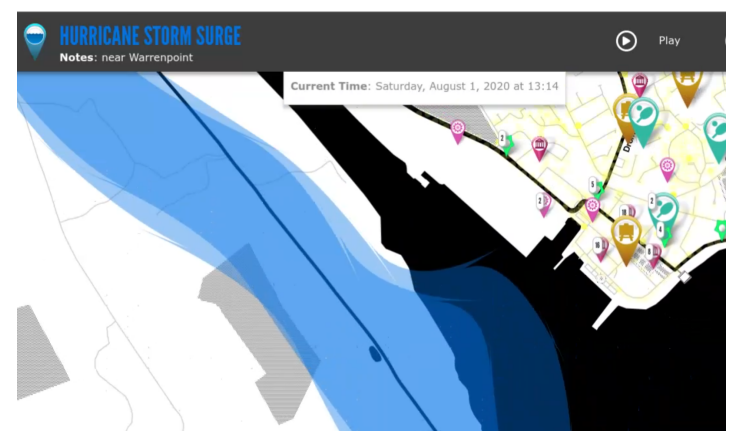

Figure 3: An ORP natural disaster simulation

\section{Planned Testing}

ORP-Sandstorms will be piloted in schools in two regions: the Eastern Province of the Kingdom of Saudi Arabia and Northern Ireland in the United Kingdom. Participants in each region will comprise students aged between 11 and 16. The KSA students will be given a storm surge simulation to experiment with while those in the UK will work with a sandstorm simulation. Participants will be organised in teams of 3 to 5 students and given simulation creation and exploration tasks with an accompanying list of activities to complete. The following is an example of the instructions to be provided.

1. Pick an area within a given location.

2. Set the natural disaster and parameters, and run the simulation.

3. Explore the timeline, and consider impacts on the community and services at various offsets from event initiation.

As a culminating activity, each team will be asked to produce one of the following:

- a poster with information warning people in their neighborhood about a pending natural disaster. 
- a podcast advising families on how to prepare for the event and assist elderly and vulnerable people.

- an emergency rescue plan for their school

\section{Conclusion and Future Work}

The ORP-Sandstorms project provides an excellent test-case for the articulated simulation approach, where both existing and new numerical models requiring not only new parameters but new datasets and algorithms, can be freely plugged in. The contrasting geographical settings of KSA and the UK, in terms of data and interface localization, introduces several technical challenges. Additionally, the platform needs to dynamically accept a new type of simulation phenomenon, sandstorms, with dynamicallydefined parameter types and numerical models, while returning data in the required schema for interface display; this is strong validation of the articulated ASC entity model and its current implementation. We are still in the process of completing the implementation and testing of the simulation. Future developments might include upgrading the simulation to incorporate virtual or augmented reality, as well as, an asynchronous chat feature that allows for communication between learners in both regions, KSA and the UK.

\section{References:}

[1] Y. Costin, M. P. O'Brien, and D. M. Slattery, Using Simulation to Develop Entrepreneurial Skills and Mind-set: An exploratory case study, Inter-national Journal of Teaching and Learning in Higher Education, Vol.30, No.1, 2018, pp. 136-145.

[2] L. Sauvé, L. Renaud, D. Kaufman, and J. S. Marquis, Distinguishing Between Games and Simulations: A systematic review, Educational Technology \& Society, Vol.10, No.3, 2007, pp. 247-256.

[3] Open Knowledge Foundation, Open Definition, http://opendefinition.org, 2020, accessed 202004-02.

[4] M. Bower, and M. S. Y. Jong, Immersive Virtual Reality in Education, Wiley Online Library, 2020.

[5] L. Vygotsky, Interaction Between Learning and Development, Readings on the Development of Children, Vol.23, No.3, 1978, pp. 34-41.

[6] D. H. Jonassen, Toward a Design Theory of Problem Solving, Educational Technology Research and Development, Vol.48, No.4, 2000, pp. 63-85.
[7] D. Laurillard, Teaching as a Design Science: Building pedagogical patterns for learning and technology, Routledge, 2013.

[8] F. A. Drews, and J. Z. Bakdash, Simulation Training in Health Care, Reviews of Human Factors and Ergonomics, Vol.8, No.1, 2013, pp. 191-234.

[9] J. Lu, P. Hallinger, and P. Showanasai, Simulation-based Learning in Management Education: A longitudinal quasi-experimental evaluation of instructional effectiveness. Journal of Management Development, Vol.33, No.3, 2014, pp. 218-244.

[10] D. Crookall, Serious Games, Debriefing, and Simulation/ Gaming as a Discipline, Simulation \&gaming, Vol.41, No.6, 2010, pp. 898-920.

[11] M. Girod, and G. R. Girod, Simulation and the Need for Practice in Teacher Preparation, Journal of Technology and Teacher Education, Vol.16, No.3, 2008, pp 307-337.

[12] P. McDermott, Building Open Government, Government Information Quarterly, Vol.27, No.4, 2010, pp. 401-413.

[13] Saudi National e-Government Program, Saudi Open Data Portal, https://www.data.gov.sa/en/, 2019, accessed: 2020-10-14.

[14] Saudi Arabia Vision 2030, National Character Enrichment Vision, https://vision2030.gov.sa/en, 2020, accessed: 2019-0820.

[15] UN.org, Number Days with Duststorm/Sandstorm, http://data.un.org/Data, 2010, accessed: 2020-10-14.

[16] L. Gonzalez and X. Briottet, North Africa and Saudi Arabia day/night sandstorm survey (NASCube), Remote Sensing, Vol. 9, No. 9, 2017, p. 896.

[17] Khalifa University, Real-Time Dust Storm Forecasting, https://www.ku.ac.ae, 2016, accessed: 2020-10-14.

[18] Data.Gov.UK, OpenDataNI, https://www.opendatani.gov.uk, 2009, accessed: 2020-10-14.

[19] M. W. AlRushaid, and A. K. J. Saudagar, Measuring the Data Openness for the Open Data in Saudi Arabia e-Government: A case study. International journal of advanced computer science and applications, Vol.7, No.12, 2016, pp. 113-122. 
[20] World Wide Web Foundation, Open Data Barometer, https://opendatabarometer.org/, 2015, accessed: 2020-10-14.

[21] OpenFaaS Ltd, OpenFaaS, https://www.openfaas.com/, 2017, accessed: 2020-10-14.

[22] Kubernetes, Kubernetes, https://kubernetes.io/, 2014, accessed: 2020-10-14.

[23] A. Logg, K. A. Mardal, and G. Wells, Eds., $A u$ tomated solution of differential equations by the finite element method: The FEniCS book (Vol. 84), Springer Science \& Business Media, 2012.

[24] D. E. J. Hobley, J. M. Adams, S. S. Nudurupati, E. W. H. Hutton, N. M. Gasparini, E. Istanbulluoglu, and G. E. Tucker GE, Creative Computing with Landlab: an Open-source Toolkit for Building, Coupling, and Exploring Two-dimensional Numerical Models of Earthsurface Dynamics, Earth Surface Dynamics, Vol.5, 2017, pp. 21-46.

[25] Weblate.org, Weblate, https://weblate.org/en/, 2012, accessed: 2020-11-14.

[26] A. A. Kononowicz, A. J. Narracott, S. Manini, M. J. Bayley, P. V. Lawford, K. McCormack, and N. Zary, A Framework for Different Levels of Integration of Computational Models into Web-based Virtual Patients, Journal of medical Internet research, Vol.16, No.1, 2014, p. e23.

[27] V. Curcin and M. Ghanem, Scientific Workflow Systems - can one size fit all?, in Proceedings of the 2008 Cairo International Biomedical Engineering Conference, Cairo, 2008, pp. 1-9.

[28] T. Oinn, Taverna: Lessons in Creating a Workflow Environment for the Life Sciences, Concurrency and Computation: Practice \& Experience, Vol. 18, No. 10, 2006, pp. 1067-1100.

[29] P. Weir, R. Ellerweg, S. Payne, D. Reuter, T. Alhonnoro, P. Voglreiter, P. Mariappan, M. Pollari, C. S. Park, P. Voigt, and T. van Oostenbrugge, Go-Smart: Open-Ended, WebBased Modelling of Minimally Invasive Cancer Treatments via a Clinical Domain Approach, arXiv preprint arXiv:1803.09166, Online, March 2018.
[30] R. Ellerweg, D. Reuter and P. Weir, Architecture of a Web-based DICOM Viewer Showing Segmentations and Simulations, 2016 IEEE 18th International Conference on e-Health Networking, Applications and Services (Healthcom), Munich, 2016, pp. 1-5.

[31] P. Mariappan, P. Weir, R. Flanagan, P. Voglreiter, T. Alhonnoro, M. Pollari, et al., GPU-based RFA Simulation for Minimally Invasive Cancer Treatment of Liver Tumours, nternational Journal of Computer Assisted Radiology and Surgery, Vol.12, No.1, 2017, pp. 59-68.

\section{Contribution of Individual Authors}

Patricia Davies designed and conducted the investigation on the educational benefits of the simulations. Phil Weir developed and implemented the ORP and ORP-Sandstorms simulation software.

Sarah Byrne was responsible for researching and collating the open datasets, and other project management activities.

\section{Acknowledgment}

Funding for this work is provided by the Prince Mohammad Bin Fahd Center for Futuristic Studies in association with the World Futures Studies Federation through the First Futures Research Grant, to whom we are deeply grateful. We thank our undergraduate Research Assistants, Fatimah Al Qahtani in KSA, and Soltanagha Huseynov in the UK, for their work on the Arabic translations and other project activities. We also thank the reviewers for the valuable feedback.

\section{Creative Commons Attribution License 4.0 (Attribution 4.0 International, CC BY 4.0)}

This article is published under the terms of the Creative Commons Attribution License 4.0

https://creativecommons.org/licenses/by/4.0/deed.en_US 